\title{
RIDGELET TRANSFORM ON SQUARE INTEGRABLE BOEHMIANS
}

\author{
RAJAKUMAR ROOPKUMAR
}

\begin{abstract}
The ridgelet transform is extended to the space of square integrable Boehmians. It is proved that the extended ridgelet transform $\mathscr{R}$ is consistent with the classical ridgelet transform $R$, linear, one-to-one, onto and both $\mathscr{R}, \mathscr{R}^{-1}$ are continuous with respect to $\delta$-convergence as well as $\Delta$-convergence.
\end{abstract}

\section{Introduction}

Motivated by the concept of Boehme's regular operators [1], the theory of Boehmians is developed in the literature. Roughly speaking, Boehmians are convolution quotients $f_{n} / \delta_{n}$ of sequences, with $\left(\delta_{n}\right)$ converges to the Dirac distribution. After the work [6] of P. Mikusiński, various Boehmian spaces have been defined and also various integral transforms have been extended on them. See $[4,5,7,9,11,12,13,16]$.

Now we recall the ridgelet transform which is introduced in $[2,15]$. Let $\mathscr{L}^{2}(\mathbb{R})$ denote the space of all complex valued Lebesgue measurable functions $\psi$ on the set $\mathbb{R}$ of all real numbers with $\int_{-\infty}^{\infty}|\psi(x)|^{2} d x<\infty, \mathscr{L}^{2}\left(\mathbb{R}^{2}\right)$ denote the space of all complex valued Lebesgue measurable functions $f$ on the set $\mathbb{R}^{2}$ with

$$
\|f\|=\left(\int_{\mathbb{R}^{2}}|f(\mathbf{x})|^{2} d \mathbf{x}\right)^{\frac{1}{2}}<\infty
$$

and $\mathscr{L}^{2}(\mathbb{Y})$ denote the space of all complex valued Lebesgue measurable functions $F$ on $\mathbb{Y}=\mathbb{R}^{+} \times \mathbb{R} \times[0,2 \pi]$ with

$$
\||| F||=\left(\int_{\mathbb{Y}}|F(a, b, \theta)|^{2} d \mu\right)^{\frac{1}{2}}<\infty,
$$

where $d \mu=d \mu(a, b, \theta)=\frac{d a}{a^{3}} d b \frac{d \theta}{4 \pi}$.

Received January 30, 2008; Revised November 3, 2008.

2000 Mathematics Subject Classification. Primary 44A15, 44A35.

Key words and phrases. Boehmians, convolution, ridgelet transform.

This work is supported by SERC Fast Track Scheme for Young Scientists from Department of Science and Technology, India. Ref. No. SR/FTP/MS-13/2006. 
For a wavelet $\psi \in \mathscr{L}^{2}(\mathbb{R})$ and $(a, b, \theta) \in \mathbb{Y}$, we define

$$
\psi_{a, b, \theta}(\mathbf{x})=a^{-\frac{1}{2}} \psi\left(\left(\mathbf{x} \cdot e^{\mathrm{i} \theta}-b\right) / a\right), \forall \mathbf{x} \in \mathbb{R}^{2} .
$$

Definition 1. The ridgelet transform of $f \in \mathscr{L}^{2}\left(\mathbb{R}^{2}\right)$ is defined by

$$
(R f)(a, b, \theta)=\int_{\mathbb{R}^{2}} f(\mathbf{x}) \psi_{a, b, \theta}(\mathbf{x}) d \mathbf{x}, \forall(a, b, \theta) \in \mathbb{Y} .
$$

It is known that $R: \mathscr{L}^{2}\left(\mathbb{R}^{2}\right) \rightarrow \mathscr{L}^{2}(\mathbb{Y})$ with the inversion formula

$$
f(\mathbf{x})=\int_{\mathbb{Y}}(R f)(a, b, \theta) \psi_{a, b, \theta}(\mathbf{x}) d \mu, \forall \mathbf{x} \in \mathbb{R}^{2}
$$

and it satisfies the Parsevel's identity $\|f\|=\|R f\| \|$ holds (see [15]).

For $f \in \mathscr{L}^{2}\left(\mathbb{R}^{2}\right)$ and $\phi \in \mathscr{D}\left(\mathbb{R}^{2}\right)$, we define the usual convolution $f * \phi$ by

$$
(f * \phi)(\mathbf{x})=\int_{\mathbb{R}^{2}} f(\mathbf{x}-\mathbf{y}) \phi(\mathbf{y}) d \mathbf{y}, \forall \mathbf{x} \in \mathbb{R}^{2} .
$$

\section{Boehmian spaces}

Boehmians are introduced as quotients of sequences to generalize the distributions. In this section, we briefly recall the construction of an abstract Boehmian space and we construct the Boehmian space

$$
\mathscr{B}_{2}\left(\mathscr{L}^{2}(\mathbb{Y}),\left(\mathscr{D}\left(\mathbb{R}^{2}\right), *\right), \star, \Delta\right)
$$

which is required to extend the ridgelet transform.

Using the notations as in [8], we let $\Gamma$ be a topological vector space, $(S, *)$ be a commutative semi group, and $\star: \Gamma \times S \rightarrow \Gamma$ is satisfying the following properties:

(1) $(f+g) \star \phi=(f \star \phi)+(g \star \phi), \forall f, g \in \Gamma$ and $\forall \phi \in S$,

(2) $(\alpha f) \star \phi=\alpha(f \star \phi), \forall \alpha \in \mathbb{C}, \forall f \in \Gamma$ and $\forall \phi \in S$,

(3) $f \star(\phi * \psi)=(f \star \phi) \star \psi, \forall f \in \Gamma$ and $\forall \phi, \psi \in S$,

(4) If $f_{n} \rightarrow f$ as $n \rightarrow \infty$ in $\Gamma$ and $\phi \in S$, then $f_{n} \star \phi \rightarrow f \star \phi$ as $n \rightarrow \infty$ in $\Gamma$,

and a collection $\Delta$ of all sequences $\left(\delta_{n}\right)$ from $S$, satisfying the following conditions:

$\left(\Delta_{1}\right):$ If $\left(\delta_{n}\right),\left(\epsilon_{n}\right) \in \Delta$, then $\left(\delta_{n} * \epsilon_{n}\right) \in \Delta$

$\left(\Delta_{2}\right)$ : If $f_{n} \rightarrow f$ as $n \rightarrow \infty$ in $\Gamma$ and $\left(\delta_{n}\right) \in \Delta$, then $f_{n} \star \delta_{n} \rightarrow f$ as $n \rightarrow \infty$ in $\Gamma$.

Every member of $\mathscr{B}=\mathscr{B}(\Gamma,(S, *), \star, \Delta)$ is of the form $\left[\frac{f_{n}}{\delta_{n}}\right]$, where $f_{n} \in$ $\Gamma, \forall n \in \mathbb{N},\left(\delta_{n}\right) \in \Delta$ and

$$
f_{n} \star \delta_{m}=f_{m} \star \delta_{n}, \forall m, n \in \mathbb{N} .
$$

Two Boehmians $\left[\frac{f_{n}}{\delta_{n}}\right],\left[\frac{g_{n}}{\phi_{n}}\right]$ in $\mathscr{B}$ are said to be equal if

$$
f_{n} \star \phi_{m}=g_{m} \star \delta_{n}, \forall m, n \in \mathbb{N} \text {. }
$$


The space $\Gamma$ is identified as a subset of $\mathscr{B}$ by the identification $f \mapsto\left[\frac{f \star \delta_{n}}{\delta_{n}}\right]$, where $\left(\delta_{n}\right) \in \Delta$ is arbitrary.

Addition, scalar multiplication and the operation $\star$ in the context of $\mathscr{B}$ are defined as follows:

$$
\begin{aligned}
{\left[\frac{f_{n}}{\delta_{n}}\right]+\left[\frac{g_{n}}{\phi_{n}}\right] } & =\left[\frac{f_{n} \star \phi_{n}+g_{n} \star \delta_{n}}{\delta_{n} * \phi_{n}}\right], \\
\alpha\left[\frac{f_{n}}{\delta_{n}}\right] & =\left[\frac{\alpha f_{n}}{\delta_{n}}\right], \\
{\left[\frac{f_{n}}{\delta_{n}}\right] \star \phi } & =\left[\frac{f_{n} \star \phi}{\delta_{n}}\right] .
\end{aligned}
$$

There are two notions of convergences on Boehmian spaces defined as follows:

Definition 2 ( $\delta$-convergence). $\left(x_{n}\right)$ is said to $\delta$-converge to $x$, denoted by $x_{n} \stackrel{\delta}{\rightarrow} x$, if there exists $\left(\delta_{k}\right) \in \Delta$ such that $x_{n} \star \delta_{k} \in \Gamma, \forall n, k \in \mathbb{N}, x \star \delta_{k} \in$ $\Gamma, \forall k \in \mathbb{N}$ and for each $k \in \mathbb{N}, x_{n} \star \delta_{k} \rightarrow x \star \delta_{k}$ as $n \rightarrow \infty$ in $\Gamma$.

Definition 3 ( $\Delta$-convergence). $\left(x_{n}\right)$ is said to $\Delta$-converge to $x$, denoted by $x_{n} \stackrel{\Delta}{\rightarrow} x$, if there exists $\left(\delta_{n}\right) \in \Delta$ such that $\left(x_{n}-x\right) \star \delta_{n} \in \Gamma, \forall n \in \mathbb{N}$ and $\left(x_{n}-x\right) \star \delta_{n} \rightarrow 0$ as $n \rightarrow \infty$ in $\Gamma$.

The following lemma gives a necessary and sufficient condition for $\delta$-convergence, which is stated and proved in [6].

Lemma 1. $\left(x_{n}\right) \stackrel{\delta}{\rightarrow} x$ as $n \rightarrow \infty$ in $\mathscr{B}$ if and only if there exist $f_{n, k}, f_{k} \in \Gamma$ and $\left(\phi_{k}\right) \in \Delta$ such that $x_{n}=\left[\frac{f_{n, k}}{\phi_{k}}\right], x=\left[\frac{f_{k}}{\phi_{k}}\right]$ and for each $k, f_{n, k} \rightarrow f_{k}$ as $n \rightarrow \infty$ in $\Gamma$.

The $\mathscr{L}^{p}$-Boehmians are constructed in [3] for $p>1$. So just we state the definition of the square integrable Boehmians. The space

$$
\mathscr{B}_{1}=\mathscr{B}\left(\mathscr{L}^{2}\left(\mathbb{R}^{2}\right),\left(\mathscr{D}\left(\mathbb{R}^{2}\right), *\right), *, \Delta\right)
$$

of square integrable Boehmians is defined by taking the topological vector space $\Gamma$ as $\mathscr{L}^{2}\left(\mathbb{R}^{2}\right)$, commutative semigroup $(S, *)$ as $\left(\mathscr{D}\left(\mathbb{R}^{2}\right), *\right)$, where $\mathscr{D}\left(\mathbb{R}^{2}\right)$ is the Schwartz testing function space consisting of smooth complex valued functions on $\mathbb{R}^{2}$ with compact supports, * is the usual convolution of functions on $\mathbb{R}^{2}$ defined by

$$
(f * \phi)(\mathbf{x})=\int_{\mathbb{R}^{2}} f(\mathbf{x}-\mathbf{y}) \phi(\mathbf{y}) d \mathbf{y},
$$

$\star$ as the same usual convolution and $\Delta$ as the collection of all sequences $\left(\delta_{n}\right)$ from $\mathscr{D}\left(\mathbb{R}^{2}\right)$ satisfying the following properties:

(1) $\int_{\mathbb{R}^{2}} \delta_{n}(\mathbf{x}) d \mathbf{x}=1$ for all $n$ in the set $\mathbb{N}$ of all natural numbers;

(2) $\int_{\mathbb{R}^{2}}\left|\delta_{n}(\mathbf{x})\right| d \mathbf{x} \leq M, \forall n \in \mathbb{N}$ for some $M>0$;

(3) Given $\epsilon>0$ there exists $m \in \mathbb{N}$ such that supp $\delta_{n} \subset B(\mathbf{0} ; \epsilon), \forall n \geq m$, where $B(\mathbf{0} ; \epsilon)=\left\{\mathbf{x} \in \mathbb{R}^{2}:|\mathbf{x}|<\epsilon\right\}$ and $|\mathbf{x}|$ is the Euclidean norm of $\mathbf{x}$ in $\mathbb{R}^{2}$. 
To construct the Boehmian space $\mathscr{B}_{2}=\mathscr{B}\left(\mathscr{L}^{2}(\mathbb{Y}),\left(\mathscr{D}\left(\mathbb{R}^{2}\right), *\right), \star, \Delta\right)$, we shall prove the following auxiliary results.

Definition 4. Let $F \in \mathscr{L}^{2}(\mathbb{Y})$ and $\phi \in \mathscr{D}\left(\mathbb{R}^{2}\right)$. For $(a, b, \theta) \in \mathbb{Y}$, we define

$$
(F \star \phi)(a, b, \theta)=\int_{\mathbb{R}^{2}} F\left(a, b-\mathbf{x} \cdot e^{\mathrm{i} \theta}, \theta\right) \phi(\mathbf{x}) d \mathbf{x} .
$$

Lemma 2. If $F \in \mathscr{L}^{2}(\mathbb{Y})$ and $\phi \in \mathscr{D}\left(\mathbb{R}^{2}\right)$, then $\|F \star \phi\||\leq C|\|F\| \mid$ for some $C>0$ and hence $F \star \phi \in \mathscr{L}^{2}(\mathbb{Y})$.

Proof. If $\phi$ is identically zero, then $F \star \phi=0$ and hence, in this case, the lemma follows. Assume that $\phi \neq 0$. If $d \nu=|\phi(x)| d x$ and $C=\int_{\mathbb{R}^{2}}|\phi(x)| d x$, then $\frac{d \nu}{C}$ is a probability measure on $\mathbb{R}^{2}$. Therefore

$$
\begin{aligned}
\|F \star \phi\|^{2} & \leq \int_{\mathbb{Y}}\left(\int_{\mathbb{R}^{2}}\left|F\left(a, b-\mathbf{x} \cdot e^{\mathrm{i} \theta}, \theta\right)\right| \cdot|\phi(\mathbf{x})| d \mathbf{x}\right)^{2} d \mu \\
& =\int_{\mathbb{Y}} C^{2}\left(\int_{\mathbb{R}^{2}}\left|F\left(a, b-\mathbf{x} \cdot e^{\mathrm{i} \theta}, \theta\right)\right| \frac{d \nu}{C}\right)^{2} d \mu
\end{aligned}
$$

(by using Jensen's inequality [14, p. 62])

$\leq \int_{\mathbb{Y}} C^{2} \int_{\mathbb{R}^{2}}\left|F\left(a, b-\mathbf{x} \cdot e^{\mathrm{i} \theta}, \theta\right)\right|^{2} \frac{d \nu}{C} d \mu$

$=C \int_{\mathbb{Y}} \int_{\mathbb{R}^{2}}\left|F\left(a, b-\mathbf{x} \cdot e^{\mathrm{i} \theta}, \theta\right)\right|^{2} \cdot|\phi(\mathbf{x})| d \mathbf{x} d \mu$

$=C \int_{\mathbb{R}^{2}} \int_{\mathbb{Y}}\left|F\left(a, b-\mathbf{x} \cdot e^{\mathrm{i} \theta}, \theta\right)\right|^{2} d \mu|\phi(\mathbf{x})| d \mathbf{x}$

(by using Fubini's theorem [14, p. 164])

$$
=C^{2}\|F\|^{2} \text {. }
$$

Hence the lemma follows.

Lemma 3. If $F_{1}, F_{2} \in \mathscr{L}^{2}(\mathbb{Y}), \phi \in \mathscr{D}\left(\mathbb{R}^{2}\right)$ and $\alpha \in \mathbb{C}$, then

(1) $\left(F_{1}+F_{2}\right) \star \phi=F_{1} \star \phi+F_{2} \star \phi$.

(2) $(\alpha F) \star \phi=\alpha(F \star \phi)$. $\int_{\mathbb{R}^{2}}$.

The proof of the lemma follows from the linearity of the integral operator

Lemma 4. If $F \in \mathscr{L}^{2}(\mathbb{Y})$ and $\phi_{1}, \phi_{2} \in \mathscr{D}\left(\mathbb{R}^{2}\right)$, then $F \star\left(\phi_{1} * \phi_{2}\right)=\left(F \star \phi_{1}\right) \star \phi_{2}$.

Proof. For $(a, b, \theta) \in \mathbb{Y}$,

$$
\begin{aligned}
\left(F \star\left(\phi_{1} * \phi_{2}\right)\right)(a, b, \theta) & =\int_{\mathbb{R}^{2}} F\left(a, b-\mathbf{x} \cdot e^{\mathrm{i} \theta}, \theta\right) \int_{\mathbb{R}^{2}} \phi_{1}(\mathbf{x}-\mathbf{y}) \phi_{2}(\mathbf{y}) d \mathbf{y} d \mathbf{x} \\
& =\int_{\mathbb{R}^{2}} \phi_{2}(\mathbf{y}) \int_{\mathbb{R}^{2}} F\left(a, b-\mathbf{x} \cdot e^{\mathrm{i} \theta}, \theta\right) \phi_{1}(\mathbf{x}-\mathbf{y}) d \mathbf{x} d \mathbf{y}
\end{aligned}
$$

(by using Fubini's theorem) 


$$
\begin{aligned}
& =\int_{\mathbb{R}^{2}} \phi_{2}(\mathbf{y}) \int_{\mathbb{R}^{2}} F\left(a, b-(\mathbf{z}+\mathbf{y}) \cdot e^{\mathrm{i} \theta}, \theta\right) \phi_{1}(\mathbf{z}) d \mathbf{z} d \mathbf{y} \\
& \quad(\text { by putting } \mathbf{z}=\mathbf{x}-\mathbf{y}) \\
& =\int_{\mathbb{R}^{2}} \phi_{2}(\mathbf{y})\left(F \star \phi_{1}\right)\left(a, b-\mathbf{y} \cdot e^{\mathrm{i} \theta}, \theta\right) d \mathbf{y} \\
& =\left(\left(F \star \phi_{1}\right) \star \phi_{2}\right)(a, b, \theta) .
\end{aligned}
$$

Lemma 5. If $\left(F_{n}\right)$ converges to $F$ in $\mathscr{L}^{2}(\mathbb{Y})$ and $\phi \in \mathscr{D}\left(\mathbb{R}^{2}\right)$, then $F_{n} \star \phi \rightarrow$ $F \star \phi$ as $n \rightarrow \infty$ in $\mathscr{L}^{2}(\mathbb{Y})$.

Proof. We have $\left\|\mid F_{n}-F\right\| \| \rightarrow$ as $n \rightarrow \infty$. In view of Lemma 2, and by using Lemma 3(1), we get

$$
\left\|| F _ { n } \star \phi - F \star \phi | \left|=\left\|\left(F_{n}-F\right) \star \phi|\|\leq C\|| F_{n}-F \mid\right\|\right.\right.
$$

for some $C>0$. Hence the lemma follows.

Lemma 6. If $F \in \mathscr{L}^{2}(\mathbb{Y})$ and $\left(\delta_{n}\right) \in \Delta$, then $F \star \delta_{n} \rightarrow F$ as $n \rightarrow \infty$ in $\mathscr{L}^{2}(\mathbb{Y})$.

Proof. Let $\epsilon>0$ be given. Since the space $C_{c}(\mathbb{Y})$ of all complex valued continuous functions on $\mathbb{Y}$ with compact supports is dense in $\mathscr{L}^{2}(\mathbb{Y})$, there exits $G \in C_{c}(\mathbb{Y})$ such that

$$
\|F-G\| \mid<\min \left\{\frac{\epsilon}{3 M}, \frac{\epsilon}{3}\right\},
$$

where $M>0$ is as in property (2) for the delta sequence $\left(\delta_{n}\right)$ (see Section 2). We note that

(3) $\quad\left\|F \star \delta_{n}-F|\|\leq\||\left|(F-G) \star \delta_{n}\right|\right\|+\left\|G \star \delta_{n}-G|\|+\|| G-F \mid\right\|$.

In view of Lemma 2 , we have

$$
\left\|(F-G) \star \delta_{n}\right\|^{2}<\||| F-G \mid\|^{2}\left(\int_{\mathbb{R}^{2}}\left|\delta_{n}(\mathbf{x})\right| d \mathbf{x}\right)^{2} \leq M^{2}\|F-G\|^{2} .
$$

Therefore

$$
\left\|(F-G) \star \delta_{n}\right\| \leq M \frac{\epsilon}{3 M}=\frac{\epsilon}{3} .
$$

Next, let $K_{1}, K_{2}$ and $K_{3}$ be compact subsets of $[0,2 \pi], \mathbb{R}$ and $\mathbb{R}^{+}$respectively, such that supp $G \subset K_{1} \times K_{2} \times K_{3}$.

Let $\mathbb{K}=K_{1} \times\left(K_{2}+[-1,1]\right) \times K_{3}$ and $C$ be such that $\int_{\mathbb{K}} d \mu<C<+\infty$. Since $G$ is uniformly continuous on $\mathbb{Y}$, we choose $0<r<1$ such that if

$$
\left(a_{1}, b_{1}, \theta_{1}\right),\left(a_{2}, b_{2}, \theta_{2}\right) \in \mathbb{Y} \text { with }\left|\left(a_{1}, b_{1}, \theta_{1}\right)-\left(a_{2}, b_{2}, \theta_{2}\right)\right|<r,
$$

then

$$
\left|G\left(a_{1}, b_{1}, \theta_{1}\right)-G\left(a_{2}, b_{2}, \theta_{2}\right)\right|<\frac{\epsilon}{3 M \sqrt{C}}
$$


where $M$ is as in the property $(2)$ of $\left(\delta_{n}\right)$ and $\left|\left(a_{1}, b_{1}, \theta_{1}\right)-\left(a_{2}, b_{2}, \theta_{2}\right)\right|$ is the Euclidean norm of $\left(a_{1}, b_{1}, \theta_{1}\right)-\left(a_{2}, b_{2}, \theta_{2}\right)$ in $\mathbb{R}^{3}$.

Let $m_{1} \in \mathbb{N}$ such that $\operatorname{supp} \delta_{n} \subset B(\mathbf{0} ; r), \forall n \geq m_{1}$. For $n \geq m_{1}$, we get

$$
\left\|G \star \delta_{n}-G\right\|^{2} \leq \int_{\mathbb{Y}}\left(\int_{\mathbb{R}^{2}}\left|\left(G\left(a, b-\mathbf{x} \cdot e^{\mathrm{i} \theta}, \theta\right)-G(a, b, \theta)\right) \delta_{n}(\mathbf{x})\right| d \mathbf{x}\right)^{2} d \mu .
$$

If $d \nu_{n}=\left|\delta_{n}(\mathbf{x})\right| d \mathbf{x}$, then it is a positive finite measure on $\mathbb{R}^{2}$ and hence $\frac{d \nu_{n}}{\sigma_{n}}$ is a probability measure on $\mathbb{R}^{2}$, where $\sigma_{n}=\int_{\mathbb{R}^{2}}\left|\delta_{n}(\mathbf{x})\right| d \mathbf{x}$. Therefore the last integral is equal to

$$
\begin{aligned}
& \int_{\mathbb{Y}} \sigma_{n}^{2}\left(\int_{\mathbb{R}^{2}}\left|G\left(a, b-\mathbf{x} \cdot e^{\mathrm{i} \theta}, \theta\right)-G(a, b, \theta)\right| \frac{d \nu_{n}}{\sigma_{n}}\right)^{2} d \mu \\
\leq & \sigma_{n}^{2} \int_{\mathbb{Y}} \int_{\mathbb{R}^{2}}\left|G\left(a, b-\mathbf{x} \cdot e^{\mathrm{i} \theta}, \theta\right)-G(a, b, \theta)\right|^{2} \frac{d \nu_{n}}{\sigma_{n}} d \mu
\end{aligned}
$$

(by using Jensen's inequality)

$$
\leq M \int_{\mathbb{Y}} \int_{B(\mathbf{0} ; r)}\left|G\left(a, b-\mathbf{x} \cdot e^{\mathrm{i} \theta}, \theta\right)-G(a, b, \theta)\right|^{2} \cdot\left|\delta_{n}(\mathbf{x})\right| d \mathbf{x} d \mu .
$$

(since $\operatorname{supp} \delta_{n} \subseteq B(\mathbf{0} ; r), n \geq m_{1}$ and $\sigma_{n} \leq M, \forall n \in \mathbb{N}$ )

Since supp $G(a, b, \theta) \subset K_{1} \times K_{2} \times K_{3}$ and $\mathbf{x} \in B(\mathbf{0} ; r)$ (with $0<r<$ $1)$, supp $G\left(a, b-\mathbf{x} \cdot e^{\mathrm{i} \theta}, \theta\right) \subset \mathbb{K}$. Therefore the last integral is equal to $M \int_{\mathbb{K}} \int_{B(\mathbf{0} ; r)}\left|G\left(a, b-\mathbf{x} \cdot e^{\mathrm{i} \theta}, \theta\right)-G(a, b, \theta)\right|^{2} \cdot\left|\delta_{n}(\mathbf{x})\right| d \mathbf{x} d \mu$. Next we observe that $\left|\left(a, b-\mathbf{x} \cdot e^{\mathrm{i} \theta}, \theta\right)-(a, b, \theta)\right|<r$. Therefore, by using the inequality (5), the last integral is dominated by

$$
M \int_{\mathbb{K}} \int_{B(\mathbf{0} ; r)}\left(\frac{\epsilon}{3 M \sqrt{C}}\right)^{2} \cdot\left|\delta_{n}(\mathbf{x})\right| d \mathbf{x} d \mu \leq M^{2} C\left(\frac{\epsilon}{3 M \sqrt{C}}\right)^{2}=\left(\frac{\epsilon}{3}\right)^{2} .
$$

Using (2), (4), and the estimate of $\left\|G \star \delta_{n}-G\right\| \|$ in (3), it follows that $\| F \star$ $\delta_{n}-F \mid \|<\epsilon$ when $n \geq m_{1}$. Hence the lemma follows.

Lemma 7. If $F_{n} \rightarrow F$ as $n \rightarrow \infty$ in $\mathscr{L}^{2}(\mathbb{Y})$ and $\left(\delta_{n}\right) \in \Delta$, then $F_{n} \star \delta_{n} \rightarrow F$ as $n \rightarrow \infty$.

Proof. We note that $\left\|F_{n} \star \delta_{n}-F\left|\|\leq\| F_{n} \star \delta_{n}-F \star \delta_{n}\|\mid+\| F \star \delta_{n}-F \|\right.\right.$. By Lemma $6,\left\|F \star \delta_{n}-F\right\| \| 0$ as $n \rightarrow \infty$. Now by Lemma 2 and the property (2) of $\left(\delta_{n}\right),\|\|\left(F_{n}-F\right) \star \delta_{n}\left\|\left|\leq M\left\|F_{n}-F \mid\right\| \rightarrow 0\right.\right.$ as $n \rightarrow \infty$.

\section{Extended ridgelet transform}

Lemma 8. If $f \in \mathscr{L}^{2}\left(\mathbb{R}^{2}\right)$ and $\phi \in \mathscr{D}\left(\mathbb{R}^{2}\right)$, then $R(f * \phi)=(R f) \star \phi$.

Proof. For $(a, b, \theta) \in \mathbb{Y}$,

$$
R(f * \phi)(a, b, \theta)=\int_{\mathbb{R}^{2}} a^{-\frac{1}{2}} \psi\left(\left(\mathbf{x} \cdot e^{\mathrm{i} \theta}-b\right) / a\right)(f * \phi)(\mathbf{x}) d \mathbf{x}
$$




$$
\begin{aligned}
& =\int_{\mathbb{R}^{2}} a^{-\frac{1}{2}} \psi\left(\left(\mathbf{x} \cdot e^{\mathrm{i} \theta}-b\right) / a\right) \int_{\mathbb{R}^{2}} f(\mathbf{x}-\mathbf{y}) \phi(\mathbf{y}) d \mathbf{y} d \mathbf{x} \\
& =\int_{\mathbb{R}^{2}} \phi(\mathbf{y}) \int_{\mathbb{R}^{2}} a^{-\frac{1}{2}} \psi\left(\left(\mathbf{x} \cdot e^{\mathrm{i} \theta}-b\right) / a\right) f(\mathbf{x}-\mathbf{y}) d \mathbf{x} d \mathbf{y} \\
& \quad \quad \text { by using Fubini's theorem }) \\
& \left.=\int_{\mathbb{R}^{2}} \phi(\mathbf{y}) \int_{\mathbb{R}^{2}} a^{-\frac{1}{2}} \psi\left((\mathbf{z}+\mathbf{y}) \cdot e^{\mathrm{i} \theta}-b\right) / a\right) f(\mathbf{z}) d \mathbf{z} d \mathbf{y} \\
& =\int_{\mathbb{R}^{2}} \phi(\mathbf{y}) \int_{\mathbb{R}^{2}} a^{-\frac{1}{2}} \psi\left(\left(\mathbf{z} \cdot e^{\mathrm{i} \theta}-\left(b-\mathbf{y} \cdot e^{\mathrm{i} \theta}\right)\right) / a\right) f(\mathbf{z}) d \mathbf{z} d \mathbf{y} \\
& =\int_{\mathbb{R}^{2}} \phi(\mathbf{y})(R f)\left(a, b-\mathbf{y} \cdot e^{\mathrm{i} \theta}, \theta\right) d \mathbf{y} \\
& =((R f) \star \phi)(a, b, \theta) .
\end{aligned}
$$

Definition 5. The extended ridgelet transform $\mathscr{R} X$ of a Boehmian $X=\left[\frac{f_{n}}{\delta_{n}}\right] \in$ $\mathscr{B}_{1}$ is defined as $\left[\frac{R f_{n}}{\delta_{n}}\right] \in \mathscr{B}_{2}$.

Lemma 9. The extended ridgelet transform is well defined.

Proof. Let $X=\left[\frac{f_{n}}{\delta_{n}}\right] \in \mathscr{B}_{1}$. Then we have $f_{n} * \delta_{m}=f_{m} * \delta_{n}, \forall m, n \in \mathbb{N}$. Applying the ridgelet transform on both sides and using the Lemma 8 , we get $\left(R f_{n}\right) \star \delta_{m}=\left(R f_{m}\right) \star \delta_{n}, \forall m, n \in \mathbb{N}$. Therefore $\frac{R f_{n}}{\delta_{n}}$ represents a Boehmian in $\mathscr{B}_{2}$. If $\frac{g_{n}}{\phi_{n}}$ is another representative of $X$, then we have $f_{n} * \phi_{m}=g_{m} *$ $\delta_{n}, \forall m, n \in \mathbb{N}$. Again applying the ridgelet transform and using Lemma 8, we get that $\left(R f_{n}\right) \star \phi_{m}=\left(R g_{m}\right) \star \delta_{n}, \forall m, n \in \mathbb{N}$. This shows that $\frac{R f_{n}}{\delta_{n}}$ and $\frac{R g_{n}}{\phi_{n}}$ represent the same Boehmian in $\mathscr{B}_{2}$. Hence the lemma follows.

Lemma 10. The ridgelet transform on $\mathscr{B}_{1}$ is consistent with the classical ridgelet transform on $\mathscr{L}^{2}(\mathbb{R})$.

Proof. Let $f \in \mathscr{L}^{2}(\mathbb{R})$. Then the Boehmian representing $f$ in $\mathscr{B}_{1}$ is $\left[\frac{f * \delta_{n}}{\delta_{n}}\right]$. It is clear that $\mathscr{R}\left[\frac{f * \delta_{n}}{\delta_{n}}\right]=\left[\frac{R\left(f * \delta_{n}\right)}{\delta_{n}}\right]=\left[\frac{R f \star \delta_{n}}{\delta_{n}}\right]$, which is the Boehmian representing the $R f$ in $\mathscr{B}_{2}$. Hence the lemma follows.

Theorem 1. The extended ridgelet transform $\mathscr{R}: \mathscr{B}_{1} \rightarrow \mathscr{B}_{2}$ is a linear map.

Proof. Let $X=\left[\frac{f_{n}}{\delta_{n}}\right], Y=\left[\frac{g_{n}}{\phi_{n}}\right] \in \mathscr{B}_{1}$ and $\alpha \in \mathbb{C}$. By using the linearity of the ridgelet transform $R$ on $\mathscr{L}^{2}\left(\mathbb{R}^{2}\right)$ and by Lemma 8 , we get

$$
\begin{aligned}
\mathscr{R}(X+Y) & =\mathscr{R}\left[\frac{f_{n} * \phi_{n}+g_{n} * \delta_{n}}{\delta_{n} * \phi_{n}}\right]=\left[\frac{R\left(f_{n} * \phi_{n}+g_{n} * \delta_{n}\right)}{\delta_{n} * \phi_{n}}\right] \\
& =\left[\frac{R\left(f_{n} * \phi_{n}\right)+R\left(g_{n} * \delta_{n}\right)}{\delta_{n} * \phi_{n}}\right]=\left[\frac{\left(R f_{n}\right) \star \phi_{n}+\left(R g_{n}\right) \star \delta_{n}}{\delta_{n} * \phi_{n}}\right] \\
& =\left[\frac{R f_{n}}{\delta_{n}}\right]+\left[\frac{R g_{n}}{\phi_{n}}\right]=\mathscr{R} X+\mathscr{R} Y .
\end{aligned}
$$


Theorem 2. The extended ridgelet transform $\mathscr{R}: \mathscr{B}_{1} \rightarrow \mathscr{B}_{2}$ is an one-to-one map.

Proof. Let $X=\left[\frac{f_{n}}{\delta_{n}}\right], Y=\left[\frac{g_{n}}{\phi_{n}}\right] \in \mathscr{B}_{1}$. If $\mathscr{R} X=\mathscr{R} Y$, then we have $\left[\frac{R f_{n}}{\delta_{n}}\right]=$ $\left[\frac{R g_{n}}{\phi_{n}}\right]$. This implies that $\left(R f_{n}\right) \star \phi_{m}=\left(R g_{m}\right) \star \delta_{n}, \forall m, n \in \mathbb{N}$. Using Lemma 8, we have $R\left(f_{n} * \phi_{m}\right)=R\left(g_{m} * \delta_{n}\right), \forall m, n \in \mathbb{N}$. Since the classical ridgelet transform $R: \mathscr{L}^{2}\left(\mathbb{R}^{2}\right) \rightarrow \mathscr{L}^{2}(\mathbb{Y})$ is one-to-one, we have $f_{n} * \phi_{m}=g_{m} * \delta_{n}$, $\forall m, n \in \mathbb{N}$. Thus we have proved that $X=Y$. Hence the lemma follows.

Theorem 3. The extended ridgelet transform $\mathscr{R}: \mathscr{B}_{1} \rightarrow \mathscr{B}_{2}$ is an onto map.

Proof. Let $H=\left[\frac{F_{n}}{\delta_{n}}\right] \in \mathscr{B}_{2}$. Then $F_{n} \in \mathscr{L}^{2}(\mathbb{Y})$ for all $n \in \mathbb{N}$. Using the inversion formula (1) of the classical ridgelet transform, if $f_{n}=R^{-1} F_{n}$ then we have

$$
f_{n}(\mathbf{x})=\int_{\mathbb{Y}} F_{n}(a, b, \theta) \psi_{a, b, \theta}(\mathbf{x}) d \mu, \forall \mathbf{x} \in \mathbb{R}^{2} .
$$

Using Lemma 8, we have for all $m, n \in \mathbb{N}, f_{n} * \delta_{m}=\left(R^{-1} F_{n}\right) * \delta_{m}=R^{-1}\left(F_{n} \star\right.$ $\left.\delta_{m}\right)=R^{-1}\left(F_{m} \star \delta_{n}\right)=\left(R^{-1} F_{m}\right) * \delta_{n}=f_{m} * \delta_{n}$. Therefore $\left[\frac{f_{n}}{\delta_{n}}\right] \in \mathscr{B}_{1}$. It is easy to verify that $\mathscr{R}\left[\frac{f_{n}}{\delta_{n}}\right]=H$. Hence the lemma follows.

In view of proof of the above lemma, one can get the inversion formula for the extended ridgelet transform, and the inverse of the extended ridgelet transform is obtained as

$$
\mathscr{R}^{-1} Y=\left[\frac{R^{-1} F_{n}}{\delta_{n}}\right] \in \mathscr{B}_{1} \text { for every } Y=\left[\frac{F_{n}}{\delta_{n}}\right] \in \mathscr{B}_{2} .
$$

Theorem 4. If $X \in \mathscr{B}_{1}, Y \in \mathscr{B}_{2}$ and $\phi \in \mathscr{D}\left(\mathbb{R}^{2}\right)$, then

(1) $\mathscr{R}(X * \phi)=\mathscr{R} X \star \phi$ and

(2) $\mathscr{R}^{-1}(Y \star \phi)=\mathscr{R}^{-1} Y * \phi$.

Proof. Let $X=\left[\frac{f_{n}}{\delta_{n}}\right]$. Then by using Lemma 8 we get, $\mathscr{R}(X * \phi)=\mathscr{R}\left[\frac{f_{n} * \phi}{\delta_{n}}\right]=$ $\left[\frac{R\left(f_{n} * \phi\right)}{\delta_{n}}\right]=\left[\frac{R f_{n} \star \phi}{\delta_{n}}\right]=\left[\frac{R f_{n}}{\delta_{n}}\right] \star \phi=\mathscr{R} X \star \phi$.

Let $Y=\left[\frac{F_{n}}{\phi_{n}}\right]$. By replacing $R f$ by $F$ and by applying $R^{-1}$ on both sides of the identity $R(f * \phi)=R f \star \phi$ in Lemma 8 , we obtain that $R^{-1} F * \phi=$ $R^{-1}(F \star \phi), \forall F \in \mathscr{L}^{2}(\mathbb{Y}), \forall \phi \in \mathscr{D}\left(\mathbb{R}^{2}\right)$. Therefore

$$
\begin{aligned}
\mathscr{R}^{-1}(Y \star \phi) & =\mathscr{R}^{-1}\left[\frac{F_{n} \star \phi}{\phi_{n}}\right]=\left[\frac{R^{-1}\left(F_{n} \star \phi\right)}{\phi_{n}}\right]=\left[\frac{R^{-1} F_{n} * \phi}{\phi_{n}}\right] \\
& =\left[\frac{R^{-1} F_{n}}{\phi_{n}}\right] * \phi=\mathscr{R}^{-1} Y * \phi .
\end{aligned}
$$

Theorem 5. The extended ridgelet transform $\mathscr{R}: \mathscr{B}_{1} \rightarrow \mathscr{B}_{2}$ and its inverse are continuous with respect to the $\delta$-convergence. 
Proof. Let $X_{n} \stackrel{\delta}{\rightarrow} X$ as $n \rightarrow \infty$ in $\mathscr{B}_{1}$. Then by Lemma 2.4 of [6], there exists $f_{n, k}, f_{k} \in \mathscr{L}^{2}\left(\mathbb{R}^{2}\right)$ and $\left(\delta_{k}\right) \in \Delta$ such that $X_{n}=\left[\frac{f_{n, k}}{\delta_{k}}\right], X=\left[\frac{f_{k}}{\delta_{k}}\right]$ and for each $k \in \mathbb{N}$,

$$
f_{n, k} \rightarrow f_{k} \text { as } n \rightarrow \infty \text { in } \mathscr{L}^{2}\left(\mathbb{R}^{2}\right) .
$$

Since the classical ridgelet transform is continuous we have

$$
R f_{n, k} \rightarrow R f_{k} \text { as } n \rightarrow \infty \text { in } \mathscr{L}^{2}(\mathbb{Y}) .
$$

Since $\mathscr{R} X_{n}=\left[\frac{R f_{n, k}}{\delta_{k}}\right], \mathscr{R} X=\left[\frac{R f_{k}}{\delta_{k}}\right]$, we get $\mathscr{R} X_{n} \stackrel{\delta}{\rightarrow} \mathscr{R} X$ as $n \rightarrow \infty$ in $\mathscr{B} 2$.

Let $Y_{n} \stackrel{\delta}{\rightarrow} Y$ as $n \rightarrow \infty$ in $\mathscr{B}_{2}$. Then there exist $F_{n, k}, F_{k} \in \mathscr{L}^{2}(\mathbb{Y})$ and $\left(\delta_{k}\right) \in \Delta$ such that $Y_{n}=\left[\frac{F_{n, k}}{\delta_{k}}\right], Y=\left[\frac{F_{k}}{\delta_{k}}\right]$ and for each $k \in \mathbb{N}$,

$$
F_{n, k} \rightarrow F_{k} \text { as } n \rightarrow \infty \text { in } \mathscr{L}^{2}(\mathbb{Y}) .
$$

Since the inverse ridgelet transform is continuous on $\mathscr{L}^{2}(\mathbb{Y})$ we have

$$
R^{-1} F_{n, k} \rightarrow R^{-1} F_{k} \text { as } n \rightarrow \infty \text { in } \mathscr{L}^{2}\left(\mathbb{R}^{2}\right) .
$$

Since $\mathscr{R}^{-1} Y_{n}=\left[\frac{R^{-1} F_{n, k}}{\delta_{k}}\right], \mathscr{R}^{-1} Y=\left[\frac{R^{-1} F_{k}}{\delta_{k}}\right]$, we get $\mathscr{R}^{-1} Y_{n} \stackrel{\delta}{\rightarrow} \mathscr{R}^{-1} Y$ as $n \rightarrow \infty$ in $\mathscr{B}_{1}$.

Theorem 6. The extended ridgelet transform $\mathscr{R}: \mathscr{B}_{1} \rightarrow \mathscr{B}_{2}$ and its inverse are continuous with respect to the $\Delta$-convergence.

Proof. Let $X_{n} \stackrel{\Delta}{\rightarrow} X$ as $n \rightarrow \infty$ in $\mathscr{B}_{1}$. Then by definition there exists $\left(\delta_{n}\right) \in \Delta$ such that $\left(X_{n}-X\right) * \delta_{n} \in \mathscr{L}^{2}\left(\mathbb{R}^{2}\right), \forall n \in \mathbb{N}$ and $\left(X_{n}-X\right) * \delta_{n} \rightarrow 0$ as $n \rightarrow \infty$ in $\mathscr{L}^{2}\left(\mathbb{R}^{2}\right)$. This means that there exist $g_{n} \in \mathscr{L}^{2}\left(\mathbb{R}^{2}\right)$ such that $\left(X_{n}-X\right) * \delta_{n}=\left[\frac{g_{n} * \delta_{k}}{\delta_{k}}\right], \forall n \in \mathbb{N}$ and $g_{n} \rightarrow 0$ as $n \rightarrow 0$ in $\mathscr{L}^{2}\left(\mathbb{R}^{2}\right)$.

Since the ridgelet transform $R: \mathscr{L}^{2}\left(\mathbb{R}^{2}\right) \rightarrow \mathscr{L}^{2}(\mathbb{Y})$ is continuous, $R g_{n} \rightarrow 0$ as $n \rightarrow 0$ in $\mathscr{L}^{2}(\mathbb{Y})$. Using Theorem 4 , we get $\left(\mathscr{R} X_{n}-\mathscr{R} X\right) \star \delta_{n}=\mathscr{R}\left(X_{n}-X\right) \star$ $\delta_{n}=\mathscr{R}\left(\left(X_{n}-X\right) * \delta_{n}\right)=\mathscr{R}\left[\frac{g_{n} * \delta_{k}}{\delta_{k}}\right]=\left[\frac{R\left(g_{n} * \delta_{k}\right)}{\delta_{k}}\right]=\left[\frac{R g_{n} * \delta_{k}}{\delta_{k}}\right], \forall n \in \mathbb{N}$. Therefore it follows that $\mathscr{R} X_{n} \stackrel{\Delta}{\rightarrow} \mathscr{R} X$ as $n \rightarrow \infty$ in $\mathscr{B}_{2}$. Hence $\mathscr{R}$ is continuous with respect to the $\Delta$-convergence.

If $Y_{n} \stackrel{\Delta}{\rightarrow} Y$ as $n \rightarrow \infty$ in $\mathscr{B}_{2}$. Then there exist $\left(\phi_{n}\right) \in \Delta$ and $G_{n} \in \mathscr{L}^{2}(\mathbb{Y})$ such that $\left(Y_{n}-Y\right) \star \phi_{n}=\left[\frac{G_{n} \star \phi_{k}}{\phi_{k}}\right], \forall n \in \mathbb{N}$ and $G_{n} \rightarrow 0$ as $n \rightarrow \infty$ in $\mathscr{L}^{2}(\mathbb{Y})$. Since $R^{-1}: \mathscr{L}^{2}(\mathbb{Y}) \rightarrow \mathscr{L}^{2}\left(\mathbb{R}^{2}\right)$ is continuous, $R^{-1} G_{n} \rightarrow 0$ as $n \rightarrow \infty$. Again by using Theorem 4 , we get $\left(\mathscr{R}^{-1} Y_{n}-\mathscr{R}^{-1} Y\right) * \phi_{n}=\mathscr{R}^{-1}\left(Y_{n}-Y\right) * \phi_{n}=$ $\mathscr{R}^{-1}\left(\left(Y_{n}-Y\right) \star \phi_{n}\right)=\mathscr{R}^{-1}\left[\frac{G_{n} \star \phi_{k}}{\phi_{k}}\right]=\left[\frac{R^{-1}\left(G_{n} \star \phi_{k}\right)}{\phi_{k}}\right]=\left[\frac{R^{-1} G_{n} * \phi_{k}}{\phi_{k}}\right]$. Thus we have proved that $\mathscr{R}^{-1}$ is continuous with respect to the $\Delta$-convergence.

Finally we observe that the space $\mathscr{B}_{1}$ is properly larger than $\mathscr{L}^{2}\left(\mathbb{R}^{2}\right)$. In deed, the example of a Boehmian not representing any distribution given in [6] can be modified to get a member of $\mathscr{B}_{1} \backslash \mathscr{L}^{2}\left(\mathbb{R}^{2}\right)$.

Acknowledgment. The author thanks the referee for his valuable suggestions and comments. 


\section{References}

[1] T. K. Boehme, The support of Mikusiński operators, Trans. Amer. Math. Soc. 176 (1973), 319-334.

[2] E. J. Candès, Harmonic analysis of neural networks, Appl. Comput. Harmon. Anal. 6 (1999), no. 2, 197-218.

[3] V. Karunakaran and N. V. Kalpakam, Hilbert transform for Boehmians, Integral Transforms, Spec. Funct. 9 (2000), no. 1, 19-36.

[4] V. Karunakaran and R. Roopkumar, Boehmians and their Hilbert transforms, Integral Transforms Spec. Funct. 13 (2002), no. 2, 131-141.

5] Ultra Boehmians and their Fourier transforms, Fract. Calc. Appl. Anal. 5 (2002), no. 2, 181-194.

[6] P. Mikusiński, Convergence of Boehmians, Jpn. J. Math. 9 (1983), no. 1, 159-179.

[7] _ Fourier transform for integrable Boehmians, Rocky Mountain J. Math. 17 (1987), no. 3, 577-582.

[8] $141-146$

[9] P. Mikusiński, A. Morse, and D. Nemzer, The two-sided Laplace transform for Boehmians, Integral Transforms Spec. Funct. 2 (1994), no. 3, 219-230.

[10] D. Nemzer, The Laplace transform on a class of Boehmians, Bull. Aust. Math. Soc. 46 (1992), no. 2, 347-352.

[11] R. Roopkumar, Wavelet analysis on a Boehmian space, Int. J. Math. Math. Sci. 2003 (2003), no. 15, 917-926.

[12] , Generalized Radon transform, Rocky Mountain J. Math. 36 (2006), no. 4, 1375-1390.

[13] _ Stieltjes transform for Boehmians, Integral Transforms Spec. Funct. 18 (2007), no. 11-12, 819-827.

[14] W. Rudin, Real and Complex Analysis, Mc. Graw Hill Inc., New York, 1987.

[15] J. L. Starck, E. J. Candès, and D. Donoho, The curvelet transform for image denoising, IEEE Trans. Image Process. 11 (2002), no. 6, 670-684.

[16] A. I. Zayed and P. Mikusiński, On the extension of the Zak transform, Methods Appl. Anal. 2 (1995), no. 2, 160-172.

Department of Mathematics

Alagappa University

KARAIKUDI-630 003, INDIA

E-mail address: roopkumarr@rediffmail.com 